\title{
Nutritional adequacy of goat milk infant formulas for term infants: a double-blind randomised controlled trial
}

\author{
Shao J. Zhou ${ }^{1,2,3}$, Thomas Sullivan ${ }^{4}$, Robert A. Gibson ${ }^{3}$, Bo Lönnerdal ${ }^{5}$, Colin G. Prosser ${ }^{6}$, \\ Dianne J. Lowry ${ }^{6}$ and Maria Makrides ${ }^{1,2,7 *}$ \\ ${ }^{1}$ Women's and Children's Health Research Institute, 72 King William Road, North Adelaide, SA 5006, Australia \\ ${ }^{2}$ Department of Paediatrics and Child Health, Flinders Medical Centre, Bedford Park, SA 5042, Australia \\ ${ }^{3}$ School of Agriculture, Food and Wine, University of Adelaide, Waite Campus, Waite Road, Urrbrae, SA 5064, Australia \\ ${ }^{4}$ Data Management and Analysis Centre, Discipline of Public Health, University of Adelaide, Adelaide, SA 5005, Australia \\ ${ }^{5}$ Department of Nutrition, University of California, Davis, CA 95616, USA \\ ${ }^{6}$ Dairy Goat Co-operative (N.Z.) Limited, Hamilton, New Zealand \\ ${ }^{7}$ School of Paediatrics and Reproductive Health, University of Adelaide, Adelaide, SA 5005, Australia \\ (Submitted 6 June 2013 - Final revision received 24 November 2013 - Accepted 2 December 2013 - First published online 6 February 2014)
}

\begin{abstract}
The safety and nutritional adequacy of goat milk infant formulas have been questioned. The primary aim of the present study was to compare the growth and nutritional status of infants fed a goat milk infant formula with those of infants fed a typical whey-based cow milk infant formula. The secondary aim was to examine a range of health- and allergy-related outcomes. A double-blind, randomised controlled trial with 200 formula-fed term infants randomly assigned to receive either goat or cow milk formula from 2 weeks to at least 4 months of age was conducted. A cohort of 101 breast-fed infants was included for comparison. Weight, length and head circumference were measured at 2 weeks and 1, 2, 3, 4, 6 and 12 months of age. Nutritional status was assessed from serum albumin, urea, creatinine, Hb, ferritin, and folate and plasma amino acid concentrations at 4 months. $Z$-scores for weight, length, head circumference and weight for length were not different between the two formula-fed groups. There were differences in the values of some amino acids and blood biomarkers between the formula-fed groups, but the mean values for biomarkers were within the normal reference range. There were no differences in the occurrence of serious adverse events, general health, and incidence of dermatitis or medically diagnosed food allergy. The incidence of parentally reported blood-stained stools was higher in the goat milk formula-fed group, although this was a secondary outcome and its importance is unclear. Goat milk formula provided growth and nutritional outcomes in infants that did not differ from those provided by a standard whey-based cow milk formula.
\end{abstract}

Key words: Infants: Growth: Breast-feeding: Formulas: Goat milk

Appropriate nutrition during infancy is important not only for normal growth and development, but also for long-term health outcomes. Breast-feeding is recommended for delivering these short- and long-term outcomes ${ }^{(1)}$. Infant formulas are used to supplement breast milk when breast milk is not sufficient or breast-feeding is not possible. Cow milk infant formulas are widely accepted as the first-line choice for healthy formula-fed infants. These are typically based on cow milk proteins from skimmed milk and have extra whey proteins added to improve the profile of essential and semi-essential amino acids ${ }^{(2,3)}$.

There is also consumer demand for goat milk infant formulas as evidenced by widespread reports of the use of raw goat milk and home-made formulas for infants ${ }^{(4-7)}$.
Goat infant formulas are manufactured in several countries. Compositional analysis of an infant formula made from goat milk without added whey proteins suggests that the amino acid profile ${ }^{(8)}$ is compatible with international standards for infant formula ${ }^{(9,10)}$. This type of goat milk formula has also been shown in animal studies to have amino acid digestibility and absorption properties similar to those of a cow infant formula with added whey ${ }^{(11)}$. Thus, it was expected that the amino acid delivery to infants would be similar for the two formulas, but this has never been tested.

In addition to meeting compositional criteria, it is important to establish the suitability and nutritional adequacy of infant formulas containing new sources of proteins through clinical trials $^{(9,12)}$. While goat milk has high-quality proteins and

Abbreviations: CIF, cow infant formula; GIF, goat infant formula.

*Corresponding author: Professor M. Makrides, fax +61 88239 0267, email maria.makrides@health.sa.gov.au 
fats and has a history of use for human nutrition in many cultures $^{(13-15)}$, there has been only one previous randomised controlled trial of infants fed a goat milk infant formula ${ }^{(16)}$. This study showed that the growth of thirty infants fed a goat milk infant formula was similar to that of thirty-two infants fed a whey-based cow milk infant formula ${ }^{(16)}$. However, the study was insufficient for assessing the safety and nutritional adequacy of goat milk formulas because it was underpowered and lacked blood biochemical data ${ }^{(17)}$.

The primary aim of the present study was to compare the growth and nutritional status of infants fed formulas based on either goat milk or cow milk in a well-powered randomised controlled trial. The secondary aim was to examine a range of health- and allergy-related outcomes, including the incidence and severity of dermatitis.

\section{Subjects and methods}

\section{Participants}

The study population included two cohorts of infants who were either fed infant formula or breast-fed at the time of recruitment. Infants were eligible for inclusion in the study if the following criteria were met: (1) a healthy term infant with gestation of 37-42 weeks and birth weight $\geq 2.5 \mathrm{~kg}$ and $\leq 4.75 \mathrm{~kg}$; (2) aged up to 2 weeks; (3) mother was exclusively feeding infant formula within 2 weeks of birth (for formula cohort) or planned to exclusively breast-feed for at least 4 months (for the breast-fed cohort). Infants were excluded if they were from multiple births or had severe congenital or metabolic disease likely to affect feeding or growth. Infants who were exclusively formula-fed or breast-fed were identified and referred by midwives in the postnatal wards at one of the following three tertiary hospitals: the Women's and Children's Hospital; the Flinders Medical Centre; the Lyell McEwin Hospital in Adelaide, Australia. The study was approved by the relevant Human Research Ethics Committees at all the three study centres. Written informed consent was obtained from all participating families. The trial was registered with the Australian New Zealand Clinical Trials Registry (ACTRN12608000047392).

\section{Nutritional composition of the study formulas}

The goat infant formula (GIF) was manufactured by Dairy Goat Co-operative (N.Z.) Limited using whole goat milk without added whey proteins (final whey:casein ratio approximately 20:80) and a blend of approximately $60 \%$ milk fat and $40 \%$ vegetable oils. The control cow infant formula (CIF) contained cow skimmed milk and whey proteins (final whey:casein ratio approximately 60:40) and vegetable oils as the source of fat and was supplied by Nutricia. The protein:energy ratio of both the study formulas was at the lower limit specified by $\operatorname{CODEX}^{(10)}$ and similar to that of the low-protein formula suggested to result in a more desired weight gain in infants ${ }^{(18)}$. The nutritional composition of both formulas is given in Table 1 .

\section{Study allocation and blinding}

Eligible formula-fed infants were randomly assigned to receive either GIF or CIF. Treatment allocation was done through a Web-based randomisation service according to a computergenerated randomisation schedule, which was prepared by an independent statistician. Stratification was by sex and study centre and used variable block sizes of 4 and 8 in equal proportions. The formulas were labelled in four different colours, two of them corresponding to GIF and the other two corresponding to CIF. Cans of both formulas were otherwise identical in appearance to maintain the blind. This ensured that neither the parents nor the research staff were aware whether the formula allocated was GIF or CIF. The blinding index was used to assess the success of blinding ${ }^{(19)}$.

\section{Study intervention}

The parents and carers of formula-fed infants were asked to feed their infants the allocated study formula from enrolment to at least 4 months of age and thereafter with other complementary foods up to 12 months of age. Study formulas were supplied free of charge until 12 months of age. For breast-feeding infants, mothers were encouraged to continue exclusive breast-feeding for about 4 to 6 months of age in line with current recommendations. Support for breast-feeding was provided by a qualified lactation consultant to mothers free of charge if needed. The timing of introduction of solids about 4 and 6 months was at the discretion of the families for both the formula-fed and breast-fed infants.

\section{Outcome assessments}

The primary outcomes were infant weight, length and head circumference, measured at enrolment, 2 weeks and 1, 2, 3, 4, 6 and 12 months. All anthropometric growth data were converted to $z$-scores using WHO Child Growth Standards (http://www. who.int/childgrowth/en/). Secondary outcomes included nutritional status, general health, tolerance to formula and allergy symptoms.

A small non-fasting blood sample $(3-5 \mathrm{ml})$ was collected to assess blood biomarkers, including $\mathrm{Hb}$, packed cell volume, serum creatinine, urea, albumin, ferritin, and folate, and plasma amino acids, at 4 months of age as indicators of general nutritional status. Fe-deficiency anaemia was defined as $\mathrm{Hb}$ concentration $<100 \mathrm{~g} / \mathrm{l}$ and ferritin concentration $<20 \mu \mathrm{g} / \mathrm{l}$ based on the diagnostic criteria of the test laboratory. $\mathrm{Hb}$ concentration was measured spectrophotometrically using a Cell Dyn 4000 analyser (Abbott Laboratories), which has a CV of less than $2 \%$. Albumin, urea and ferritin concentrations were measured using the Cobas/Hitachi Cobas C System, Cobas 6000 automated analyser (Roche Diagnostics). Albumin concentration was determined spectrophotometrically by an end-point bromocresol green dye-binding method. Urea concentration was measured spectrophotometrically by an enzymatic method. The test method used for measuring ferritin concentration was particle-enhanced immunoturbidmetry. The method used for measuring albumin and urea concentrations 
Table 1. Nutritional composition of the two infant formulas used in the study

\begin{tabular}{|c|c|c|c|}
\hline Nutrients & $\begin{array}{l}\text { Goat milk formula } \\
\text { (per } 418 \mathrm{~kJ}(100 \mathrm{kcal}))\end{array}$ & $\begin{array}{l}\text { Cow milk formula } \\
\text { (per } 418 \mathrm{~kJ}(100 \mathrm{kcal}))\end{array}$ & $\begin{array}{l}\text { Mature human milk } \\
\quad(\text { per } 100 \mathrm{~g})\end{array}$ \\
\hline \multicolumn{4}{|l|}{ Energyt } \\
\hline kcal & $65 \cdot 6$ & 64.8 & 70 \\
\hline $\mathrm{kJ}$ & 274.0 & 271.0 & 291 \\
\hline Protein $(\mathrm{g})$ & $2 \cdot 0$ & $2 \cdot 1$ & 1.0 \\
\hline Fat $(g)$ & $5 \cdot 3$ & $5 \cdot 2$ & 4.4 \\
\hline Saturated fat $(\mathrm{g})$ & $2 \cdot 0$ & 2.0 & - \\
\hline Unsaturated fat $(\mathrm{g})$ & 3.3 & 3.2 & - \\
\hline Linoleic acid $(n-6)(\mathrm{g})$ & 0.6 & 0.9 & - \\
\hline$\alpha$-Linolenic acid $(n-3)(\mathrm{g})$ & 0.1 & 0.1 & _- \\
\hline Carbohydrate (g) & 11.0 & 11.0 & 6.9 \\
\hline \multicolumn{4}{|l|}{ Vitamins } \\
\hline Vitamin A (RE) $(\mu \mathrm{g})$ & $141 \cdot 0$ & $87 \cdot 0$ & 61 \\
\hline Vitamin $D_{3}(\mu \mathrm{g})$ & 1.8 & $2 \cdot 1$ & 0.1 \\
\hline Vitamin E (TE) (mg) & $2 \cdot 6$ & $1 \cdot 1$ & 0.08 \\
\hline Vitamin $K_{1}(\mu \mathrm{g})$ & $12 \cdot 0$ & 8.8 & - \\
\hline Vitamin $\mathrm{C}(\mathrm{mg})$ & $20 \cdot 0$ & $12 \cdot 0$ & 5 \\
\hline Thiamin $(\mu \mathrm{g})$ & $118 \cdot 0$ & 58.0 & 10 \\
\hline Riboflavin $(\mu \mathrm{g})$ & $226 \cdot 0$ & $250 \cdot 0$ & 40 \\
\hline Niacin $(\mathrm{mg})$ & 1.3 & 0.8 & 0.18 \\
\hline Vitamin $B_{6}(\mu \mathrm{g})$ & $80 \cdot 0$ & $65 \cdot 0$ & - \\
\hline Folic acid $(\mu \mathrm{g})$ & $12 \cdot 0$ & 21.0 & $5.0 \ddagger$ \\
\hline Pantothenic acid (mg) & 0.6 & 1.2 & 0.22 \\
\hline Vitamin $B_{12}(\mu \mathrm{g})$ & 0.3 & 0.5 & 0.05 \\
\hline Biotin $(\mu \mathrm{g})$ & 3.8 & 4.7 & - \\
\hline \multicolumn{4}{|l|}{ Minerals } \\
\hline $\mathrm{Ca}(\mathrm{mg})$ & 98.0 & 81.0 & 32 \\
\hline$P(\mathrm{mg})$ & 73.0 & 53.0 & 14 \\
\hline $\mathrm{Na}(\mathrm{mg})$ & 31.0 & 31.0 & 17 \\
\hline $\mathrm{K}(\mathrm{mg})$ & 133.0 & $116 \cdot 0$ & 51 \\
\hline $\mathrm{Cl}(\mathrm{mg})$ & $116 \cdot 0$ & 71.0 & - \\
\hline $\mathrm{Mg}(\mathrm{mg})$ & $10 \cdot 0$ & $10 \cdot 0$ & 3 \\
\hline $\mathrm{Fe}(\mathrm{mg})$ & 1.0 & 1.3 & Trace \\
\hline $\mathrm{Zn}(\mathrm{mg})$ & 0.9 & 0.7 & 0.2 \\
\hline $\mathrm{I}(\mu \mathrm{g})$ & $15 \cdot 0$ & $17 \cdot 0$ & - \\
\hline $\mathrm{Cu}(\mu \mathrm{g})$ & 76.0 & 70.0 & 0.1 \\
\hline $\mathrm{Mn}(\mu \mathrm{g})$ & $16 \cdot 0$ & $12 \cdot 0$ & - \\
\hline $\mathrm{Se}(\mu \mathrm{g})$ & 1.9 & 3.7 & 1.8 \\
\hline Inositol (mg) & $6 \cdot 8$ & $5 \cdot 1$ & - \\
\hline Choline (mg) & $27 \cdot 0$ & $19 \cdot 0$ & - \\
\hline Taurine (mg) & 8.9 & 6.6 & - \\
\hline Carnitine $(\mathrm{mg})$ & 1.2 & $3 \cdot 3$ & - \\
\hline
\end{tabular}

$R E$, retinol equivalents; TE, $\alpha$-tocopherol equivalents.

* Wijesinha-Bettoni \& Burlingame ${ }^{(32)}$.

†The energy content was calculated based on $14 \mathrm{~g}$ powder added to $100 \mathrm{ml}$ water. $\ddagger$ Folate.

has a CV of less than $3 \%$ and that used for ferritin has a CV of less than $4 \%$. Serum folate concentration was determined with the ARCHITECT $i$ optical system (Abbott) using the Chemiluminescent Microparticle Immunoassay Technology, and this method has a $\mathrm{CV}$ of less than $4 \%$. Amino acid concentrations were measured on the Hitachi L-8900 Amino Acid Analyser. Plasma samples $(200 \mu \mathrm{l})$ were acidified with $50 \mu \mathrm{l}$ sulphosalicyclic acid to precipitate intact proteins before analysis. The supernatant was mixed with lithium diluent spiked with S-2-aminoethyl-L-cysteine. The L-8900 Hitachi Analyser utilises a lithium citrate buffer system and ion-exchange (Hitachi column) chromatography to separate amino acids followed by 'post-column' ninhydrin reaction detection.

At each growth assessment time point, parents/carers were asked through a structured interview whether their infants had experienced any health problems including respiratory illness, gastrointestinal illness, reflux, eye infection, ear, nose and throat conditions, fever, urinary tract infection and thrush. Serious adverse events, defined as death or hospital admission for more than $24 \mathrm{~h}$ during the 12 -month study period, were also recorded.

At the same time of growth assessments, the incidence and severity of dermatitis were assessed by trained research staff using SCORAD ${ }^{(20)}$. Food allergy was diagnosed by medical practitioners. Parents/carers were also asked whether their infants had any symptoms related to food allergy and/or gastrointestinal function including hives, swelling of the face or body, wheeze/stridor, vomiting, loose watery stools, blood-stained stools and itchy rash.

Parents/carers were asked to assess stool frequency, consistency and effort as indicators of tolerance to formula using the Bristol Stool Scale ${ }^{(21)}$ as a guide. Sleeping patterns including length of each sleep episode, total number of sleep episodes during the day, and time taken to settle for 
sleep during the day, in the evening or at night were also assessed by parental report based on the sleep and Settle Questionnaire $^{(22)}$.

\section{Other assessments}

Demographic and baseline characteristics, including infant sex, weight and length at birth, age at enrolment, and anthropometric measurements at enrolment and maternal age, BMI, parity, and history of smoking and drug and alcohol use during pregnancy, were recorded at trial entry.

\section{Sample size and power calculation}

Sample size calculations estimated that sixty-four infants per group were required to detect a 0.5 sD difference ( $80 \%$ power with $\alpha=0.05)$ in weight ${ }^{(12)}$. We aimed to enrol 100 infants per feeding group and 100 breast-fed infants to provide reference data. This sample size was also sufficient to detect a clinically important difference of 0.11 (SD 0.26) $\mathrm{g} / \mathrm{l}$ in serum albumin, an indicator of protein adequacy, with $80 \%$ power $(\alpha=0.05)$.

\section{Statistical analyses}

All analyses were carried out using SAS ${ }^{\circledR}$ software version 9.2 or a later version (SAS Institute, Inc.). Blinded treatment codes were included in the database, and analyses of the primary and secondary outcomes were carried out blinded to treatment group. All analyses were carried out using both intention-to-treat and per-protocol approaches, with infants who did not complete the trial or who had consumed any non-study formula, liquids or solids for more than $12 \mathrm{~d}$ between 2 weeks and 4 months of age being excluded from the per-protocol analysis. As the two analysis approaches yielded similar results, only results of the primary intentionto-treat analysis are reported herein.

To minimise bias in the estimation of treatment effects due to missing data, multiple imputation was used to create fifty complete datasets for analysis. The parametric regression method was used to impute continuous variables and the logistic regression method was used for binary variables. In addition to the primary imputed analysis, sensitivity analyses were carried out on the original data and on imputed data created using different seeds and using different imputation models. All approaches yielded similar results; thus, only the results of the primary imputed analysis are reported herein.

Continuous outcomes measured at multiple assessments, including the primary anthropometric outcomes, were compared between the formula-fed and breast-fed groups over time using linear mixed-effects models. Fixed effects for group, time and the interaction between group and time were included in the models, while dependence was accounted for by allowing for correlated residuals within a child. Independent of the statistical significance of the interaction term, differences between the groups were reported separately at each time point, with the effects of treatment group being expressed as mean differences. Continuous outcomes measured at a single time point were compared between the groups using linear regression models, with the effects of group being expressed as mean differences. Binary outcomes were analysed using log binomial regression models, with the effects of group being expressed as relative risks. Rare binary outcomes were analysed using Fisher's exact tests. Both unadjusted and adjusted analyses were carried out, with conclusions on group differences being based on the adjusted analyses. For the primary growth outcomes, comparisons of the two randomised groups were adjusted for centre, while comparisons involving the breast-fed reference group were adjusted for maternal education and the relevant anthropometric $z$-score at birth. All secondary outcomes were adjusted for the stratification variables centre and sex for comparisons of the randomised groups and maternal education and birth weight for comparisons involving the breast-fed reference group. Due to imbalances in maternal smoking during pregnancy between the randomised groups, sensitivity analyses of the primary growth outcomes adjusting for centre and maternal smoking during pregnancy were also carried out. All tests were two tailed with a significance level of $P \leq 0.05$.

\section{Results}

The participants were recruited between April 2008 and April 2009 from three tertiary hospitals in Adelaide. Of the 1180 families who were approached to participate in the study, 768 were eligible and 301 (39\%) consented. A total of 200 infants were formula-fed and 101 were breast-fed. More details are given in the flow chart of study participant selection in Fig. 1.

Maternal characteristics as well as infant anthropometrics at birth and at study entry are summarised in Table 2 . The mean age of infants at study entry was 6.2 (SD 3.7) $\mathrm{d}$ and $46 \%$ were male. The baseline characteristics of the participants were comparable between the two formula-fed groups, with the exception that the percentage of mothers who smoked during pregnancy was higher in the GIF group (45\%) than in the CIF group (34\%). Compared with the formula-fed infants, the reference group of breast-fed infants had a higher mean birth weight $(P=0.001)$, a lower mean maternal pre-pregnancy BMI $(P<0 \cdot 0001)$, a lower percentage of mothers who smoked $(P<0 \cdot 0001)$ during pregnancy and a higher percentage of parents who completed higher education $(P<0 \cdot 0001)$. The percentage of mothers who did not know their baby's treatment group was similar between the groups (32\% in the GIF group and $34 \%$ in the CIF group). The blindness index, which indicates the percentage of mothers who guessed their treatment group correctly above chance, was $3.8 \%$ for the GIF group compared with $2 \cdot 7 \%$ for the CIF group.

The median daily intake of study formula ranged from 698 (interquartile range $570-825$ ) $\mathrm{ml}$ in the first 2 weeks to 1000 (interquartile range $855-1190) \mathrm{ml}$ at 4 and 6 months. Compliance with the definition of exclusive formula feeding or breast-feeding ${ }^{(23)}$ from enrolment to 4 months of age was observed in seventy-six $(75 \%)$ of the 101 infants in the breast-fed group, seventy-four (73\%) of the 101 infants in 


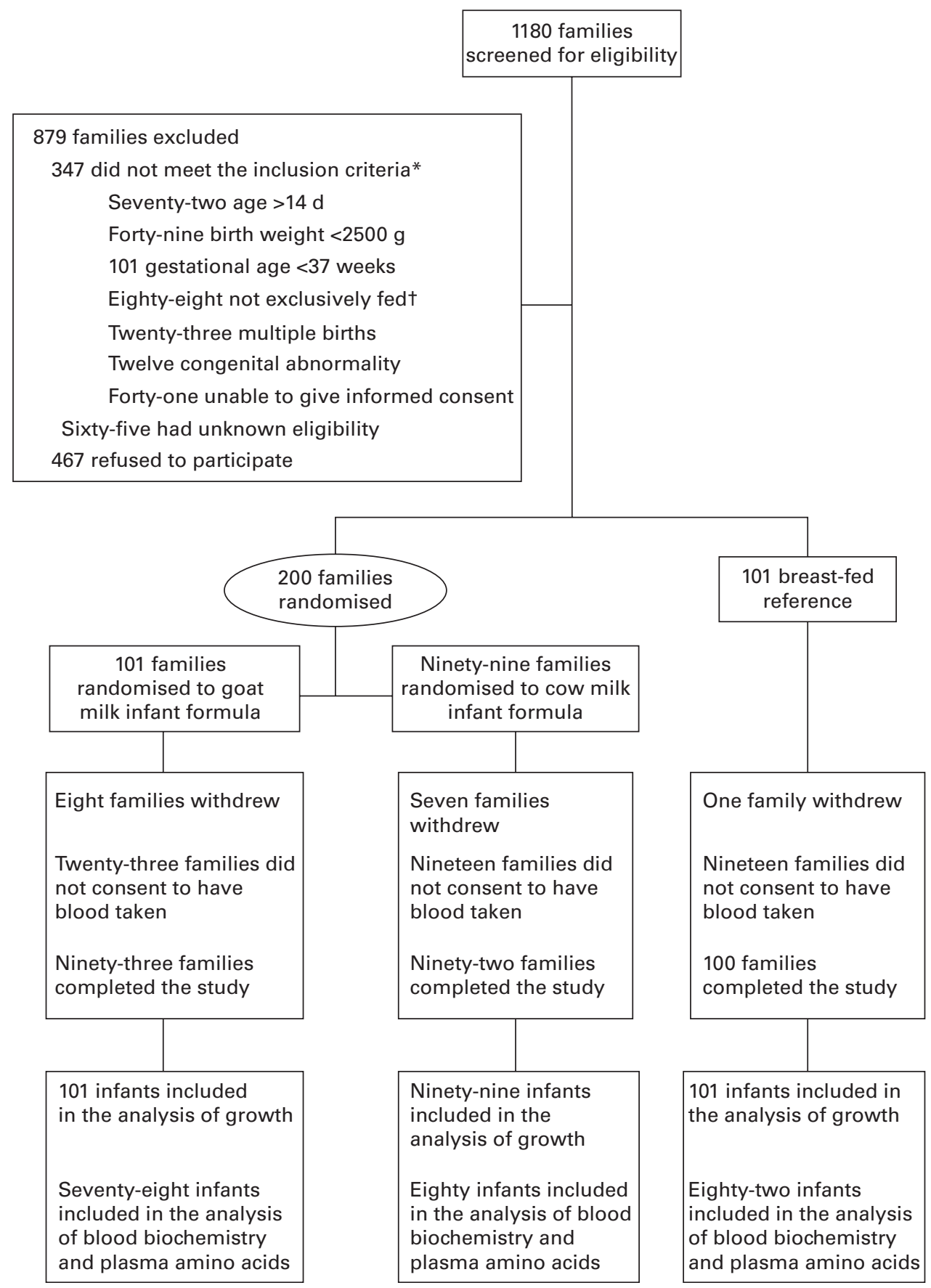

Fig. 1. Flow chart of study participants. ${ }^{*}$ Infants could be ineligible for more than one reason. †Either formula or breast milk.

the GIF group and fifty-nine $(60 \%)$ of the ninety-nine infants in the CIF group. The level of compliance in the GIF group was significantly different from that in the CIF group $(P=0.02)$, but not significantly different from that in the breast-fed reference group $(P=0 \cdot 37)$.

\section{Growth}

There were no differences in the adjusted intention-to-treat analyses of weight, length, head circumference and weightfor-length $z$-scores between the two formula-fed groups over the 12-month study period (Fig. 2(a)-(d), respectively), with or without adjustment for baseline difference in maternal smoking. Also, gains in weight, length or head circumference from registration to 4 or 6 months did not differ between the two formula-fed groups (data not shown).

In comparison with the breast-fed infants, infants in the GIF group had higher weight $z$-scores at 3, 4 and 6 months (mean difference $0.22(P=0.04), 0.30(P=0.005)$ and $0.33(P=0.003))$, while infants in the CIF group had higher weight $z$-scores from 2 to 12 months of age (mean difference $0.22(P=0.04)$, $0.28(P=0.01), 0.39(P=0.001), 0.38(P=0.001)$ and 0.36 
Table 2. Characteristics of the participants

(Mean values and standard deviations; number of participants and percentages)

\begin{tabular}{|c|c|c|c|c|c|c|c|}
\hline & \multicolumn{2}{|c|}{$\operatorname{GIF}(n 101)$} & \multicolumn{2}{|c|}{ CIF ( $n$ 99) } & \multicolumn{2}{|c|}{$\mathrm{BF}(n 101)$} & \multirow[b]{2}{*}{$P^{*}(\mathrm{FF} v . \mathrm{BF})$} \\
\hline & Mean & SD & Mean & SD & Mean & SD & \\
\hline \multicolumn{8}{|l|}{ Maternal characteristics } \\
\hline Age (years) & $27 \cdot 8$ & $6 \cdot 6$ & $28 \cdot 2$ & 5.8 & $30 \cdot 7$ & $5 \cdot 2$ & 0.0002 \\
\hline Race, Caucasian & \multirow{2}{*}{\multicolumn{2}{|c|}{92}} & & & & & \\
\hline$n$ & & & \multicolumn{2}{|c|}{94} & \multicolumn{2}{|c|}{93} & \\
\hline$\%$ & \multicolumn{2}{|c|}{91} & \multicolumn{2}{|c|}{95} & \multicolumn{2}{|c|}{92} & \\
\hline Education & & & & & & & $<0.0001$ \\
\hline \multicolumn{8}{|l|}{ Secondary incomplete } \\
\hline$n$ & \multicolumn{2}{|c|}{30} & \multicolumn{2}{|c|}{36} & \multicolumn{2}{|c|}{10} & \\
\hline$\%$ & \multicolumn{2}{|c|}{30} & \multicolumn{2}{|c|}{36} & \multicolumn{2}{|c|}{10} & \\
\hline \multicolumn{8}{|c|}{ Certificate/diploma or secondary complete } \\
\hline$n$ & \multicolumn{2}{|c|}{65} & \multicolumn{2}{|c|}{58} & \multicolumn{2}{|c|}{50} & \\
\hline$\%$ & \multicolumn{2}{|c|}{64} & \multicolumn{2}{|c|}{59} & \multicolumn{2}{|c|}{50} & \\
\hline \multicolumn{8}{|l|}{ Degree or higher degree } \\
\hline$n$ & \multicolumn{2}{|c|}{6} & \multicolumn{2}{|c|}{5} & & & \\
\hline$\%$ & & & & & & & \\
\hline $\mathrm{BMI}\left(\mathrm{kg} / \mathrm{m}^{2}\right)$ & $26 \cdot 6$ & $6 \cdot 3$ & $27 \cdot 8$ & $7 \cdot 6$ & $24 \cdot 6$ & 4.5 & 0.0007 \\
\hline Smoking during pregnancy & & & & & & & \\
\hline$n$ & & & & & & & $<0.0001$ \\
\hline$\%$ & & & & & & & \\
\hline Infant birth characteristics & & & & & & & \\
\hline Sex, male & & & & & & & \\
\hline$n$ & & & & & & & 0.63 \\
\hline$\%$ & & & & & & & \\
\hline GA at birth (weeks) & 39.4 & 1.0 & $39 \cdot 3$ & $1 \cdot 1$ & $39 \cdot 6$ & $1 \cdot 0$ & 0.048 \\
\hline Birth weight (g) & 3379 & 466 & 3407 & 419 & 3564 & 409 & 0.001 \\
\hline Birth length $(\mathrm{cm})$ & 49.5 & $2 \cdot 0$ & $49 \cdot 3$ & $2 \cdot 1$ & $50 \cdot 2$ & $2 \cdot 0$ & 0.003 \\
\hline Birth head circumference $(\mathrm{cm})$ & $34 \cdot 7$ & 1.4 & 34.6 & 1.5 & $35 \cdot 1$ & 1.2 & 0.01 \\
\hline Infant baseline data & & & & & & & \\
\hline Age at enrolment (d) & $6 \cdot 0$ & $3 \cdot 6$ & $6 \cdot 1$ & 3.7 & $6 \cdot 5$ & 3.8 & 0.35 \\
\hline Weight at enrolment $(\mathrm{g})$ & 3345 & 452 & 3371 & 423 & 3491 & 447 & 0.01 \\
\hline Length at enrolment (cm) & $50 \cdot 0$ & $2 \cdot 0$ & 49.9 & $2 \cdot 1$ & $50 \cdot 9$ & $2 \cdot 0$ & 0.0001 \\
\hline Head circumference at enrolment $(\mathrm{cm})$ & 35.0 & $1 \cdot 2$ & $35 \cdot 1$ & 1.4 & 35.5 & 1.3 & 0.009 \\
\hline
\end{tabular}

GIF, goat milk infant formula; CIF, cow milk infant formula; BF, breast-fed; FF, formula-fed; GA, gestational age.

${ }^{\star}$ Continuous and categorical characteristics compared using independent-samples $t$ tests and $\chi^{2}$ tests, respectively.

$(P=0.001))$. Infants in the GIF group had lower length $z$-scores at 2 weeks and 1 month of age compared with the breast-fed infants (mean difference $-0.33(P=0.003)$ and $-0.37(P=0.001))$, whereas those in the CIF group had higher length $z$-scores at 4, 6 and 12 months of age (mean difference $0.25 \quad(P=0.03), \quad 0.35 \quad(P=0.002)$ and 0.25 $(P=0.03)$ ). While infants in the GIF and breast-fed groups had similar head circumference $z$-scores, those in the CIF group had higher $z$-scores at 2 and 6 months of age compared with the breast-fed infants (mean difference $0 \cdot 24(P=0 \cdot 04)$ and $0.3(P=0 \cdot 01)$ ). Infants in the GIF group had higher weight-forlength $z$-scores compared with the breast-fed infants at 1 month of age only (mean difference $0.40 \quad(P=0.004)$ ), while those in the CIF group had higher weight-for-length $z$-scores at 1 and 2 months (mean difference $0.46(P=0.001)$ and $0.39(P=0.006))$. There were no statistically significant differences between the formula-fed and breast-fed groups at any other time points.

\section{Biomarkers of nutritional status}

There were no differences in serum albumin, $\mathrm{Hb}$, packed cell volume and ferritin values between the two formula-fed groups. No infants in either formula-fed group had Fe-deficiency anaemia (defined as $\mathrm{Hb}$ concentration $<100 \mathrm{~g} / \mathrm{l}$ and ferritin concentration $<20 \mu \mathrm{g} / \mathrm{l}$ ). Infants in the GIF group had lower mean serum urea, creatinine and folate concentrations compared with those in the CIF group (Table 3). Compared with the breast-fed infants, formula-fed infants had higher mean serum urea concentrations, infants in the GIF group had lower mean serum folate concentrations and infants in the CIF group had higher mean folate concentrations (Table 3). Mean serum folate concentrations in all the three groups of infants were within the normal reference range for infants of this age ${ }^{(24)}$.

The concentrations of essential and semi-essential amino acids in the plasma of infants are presented in Fig. 3. The concentrations of valine and phenylalanine were higher and those of isoleucine and threonine were lower in the plasma of infants fed the GIF than in that of infants fed the CIF. The mean difference for valine was 37 (95\% CI 25, 50) $\mu \mathrm{g} / \mathrm{l}$, phenylalanine $5(95 \% \mathrm{CI} 0,10) \mu \mathrm{g} / \mathrm{l}$, isoleucine -9 (95\% CI $-16,-3) \mu \mathrm{g} / \mathrm{l}$ and threonine -32 (95\% CI - 45, - 18) $\mu \mathrm{g} / \mathrm{l}$. The concentrations of all the other essential and semi-essential amino acids in the plasma of formula-fed infants did not differ significantly between the groups.

Compared with the breast-fed infants, infants fed the GIF had significantly higher concentrations of lysine, methionine, phenylalanine, threonine and valine. Mean differences were 
(a)

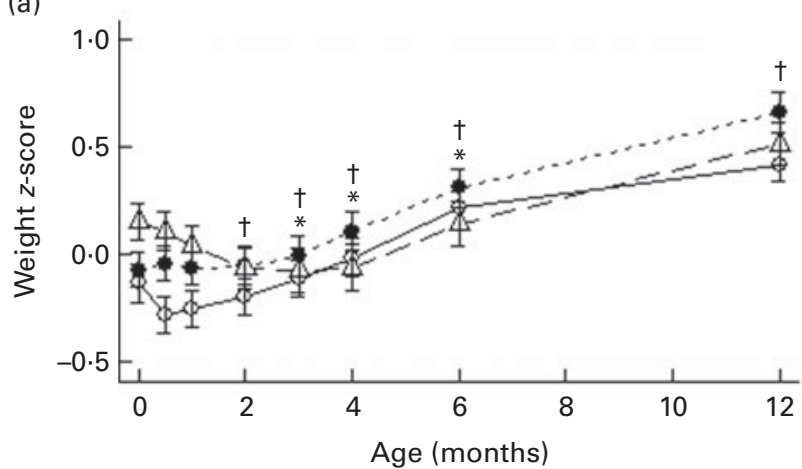

(c)

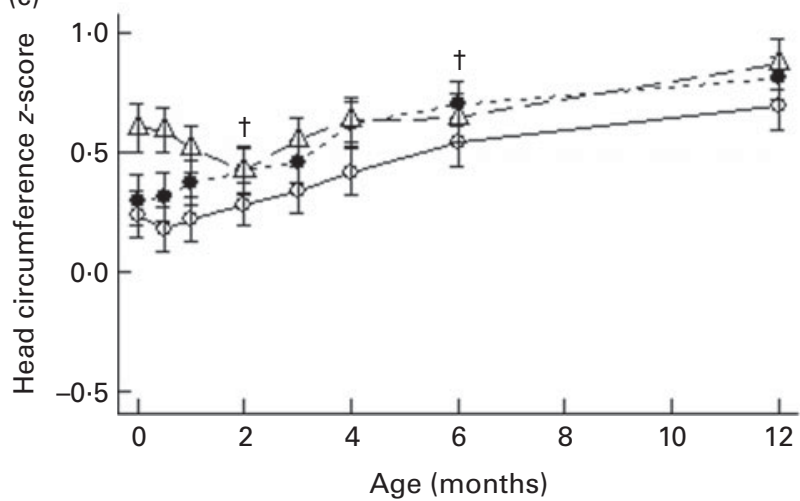

(b) 1.0 -

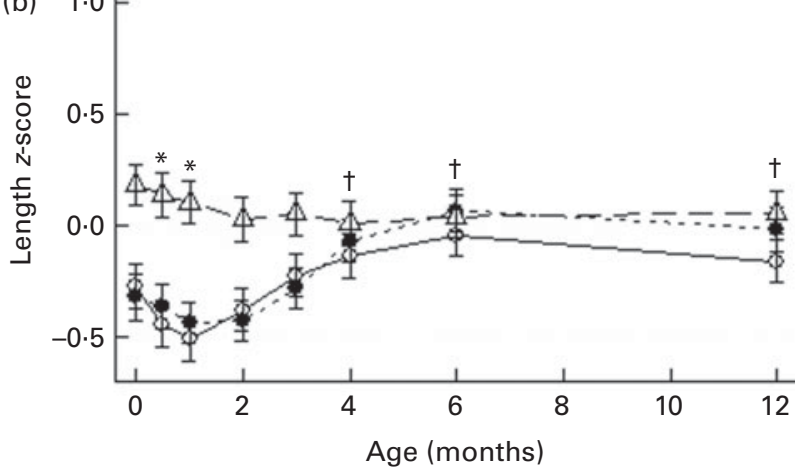

(d)

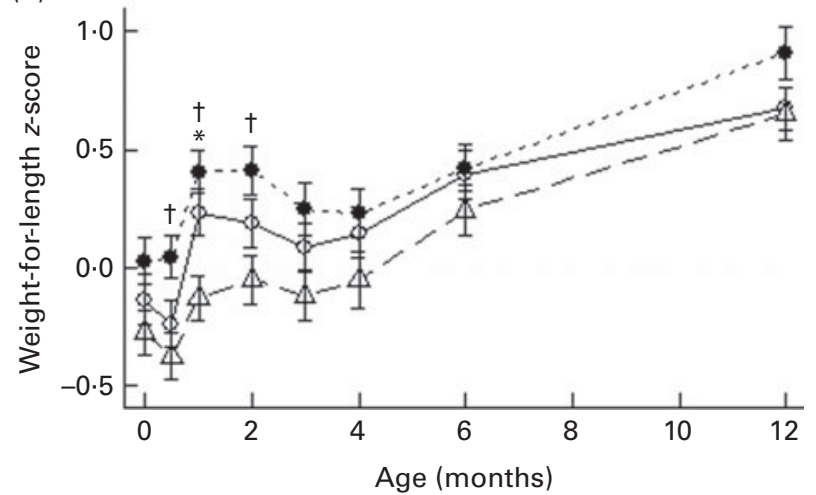

Fig. 2. Weight (a), length (b), head circumference (c) and weight-for-length (d) $z$-scores of infants fed goat milk formula $(O)$, cow milk formula $(\bullet)$ or breast milk $(\Delta)$. $Z$-score data were based on WHO reference data. Values are means of imputed data, with standard deviations represented by vertical bars. ${ }^{*}$ Mean value of the goat formula-fed group was significantly different from that of the breast milk-fed group $(P<0.05)$. † Mean value of the cow formula-fed group was significantly different from that of the breast milk-fed group $(P<0.05)$.

$15(95 \%$ CI 1, 29) $\mu \mathrm{g} / 1,6(95 \%$ CI 4, 9) $\mu \mathrm{g} / 1,13(95 \%$ CI 7 , 18) $\mu \mathrm{g} / 1,13(95 \% \mathrm{CI} 7,18) \mu \mathrm{g} / 1,19(95 \% \mathrm{CI} 4,34) \mu \mathrm{g} / 1$ and 66 (95\% CI 52, 79) $\mu \mathrm{g} / 1$, respectively. The concentrations of isoleucine, leucine, lysine, methionine, phenylalanine, threonine and valine were higher in the plasma of infants fed the CIF than in that of the breast-fed infants. Mean differences were $13(95 \%$ CI 7, 20) $\mu \mathrm{g} / 1,11(95 \%$ CI 2, 21) $\mu \mathrm{g} / 1,19(95 \%$ CI 6, 33) $\mu \mathrm{g} / 1,6$ (95\% CI 3, 8) $\mu \mathrm{g} / 1,8$ (95\% CI 2, 13) $\mu \mathrm{g} / 1,51$ (95\% CI 37, 66) $\mu \mathrm{g} / \mathrm{l}$ and $29(95 \% \mathrm{CI} 15,44) \mu \mathrm{g} / \mathrm{l}$, respectively. The concentrations of none of the amino acids were lower in either formula-fed group compared with those in the breast-fed infants.

\section{General health- and allergy-related outcomes}

There were no differences in the risk of an adverse health condition, including respiratory illness, gastrointestinal illness, reflux, eye infection, ear, nose and throat conditions, fever, urinary tract infection and thrush, between the two formulafed groups. There were also no differences in the risk of the above-mentioned health conditions between the formula-fed groups and the breast-fed reference group, with the exception that more infants had oral thrush in the CIF group than in the breast-fed reference group ( $n$ 9/86 v. $n$ 2/99, $P=0.02$ ) during the 12-month study period. The proportion of infants who had any serious adverse events during the 12-month study period was similar between the GIF, CIF and breast-fed reference groups: $n$ 15/101 (14.9\%); $n$ 12/99 (12.1\%); $n$ 9/101
(8.9\%), respectively $(P=0.43)$. The most common serious adverse events were bronchiolitis and other respiratory infections. No infants died.

The proportions of infants with medically diagnosed food allergy (GIF $n$ 2/92 $v$. CIF $n$ 1/89 $v$. breast-fed $n$ 5/99) or dermatitis assessed using SCORAD (GIF $n$ 13/91 $v$. CIF $n$ 20/86 $v$. breast-fed $n$ 21/99) did not differ between the groups. The mean SCORAD score of infants with dermatitis was 9.9 (SD 6.7) for the GIF group, 11.9 (SD 7.1) for the CIF group and 11.1 (SD 6.3) for the breast-fed group.

There was no difference between the formula-fed groups with regard to the proportion of infants with parentally reported symptoms related to allergy and/or gastrointestinal function, except for parentally reported blood-stained stools (Table 4). Compared with the breast-fed infants, infants in the GIF group had a higher risk of blood-stained stools, while infants in the CIF group had a higher risk of wheeze (Table 4). The proportions of infants with hives (GIF $n 5 / 89$ $v$. CIF $n 5 / 86 v$. breast-fed $n$ 6/99) and swelling of the face (GIF $n 6 / 89 v, n 6 / 86 v$. breast-fed $n$ 5/99) did not differ between all the groups in simple unadjusted analyses.

\section{Tolerance to formula}

The mean numbers of stool motions per $\mathrm{d}$ in infants in the GIF group at 2 weeks, 1 month, 2 months and 3 months of age were 2.5 (SD 1.6), 2.0 (sD 1.3), 1.6 (SD 1.0) and 1.6 (SD 0.9), 
respectively. These values were not different from those in infants in the CIF group, which were 2.5 (SD 1.4), $2 \cdot 0$ (SD 1.4), 1.5 (SD 0.9) and 1.6 (SD 1.3) at 2 weeks, 1 month, 2 months and 3 months, respectively. However, stool frequency in both the formula-fed groups was significantly lower $(P<0.001)$ than that in the breast-fed group (6.3 (sD 3.3), 5.0 (sD 2.3), 3.0 (SD 2.2) and 2.4 (SD 1.8) at 2 weeks, 1 month, 2 months and 3 months, respectively). Compared with infants in the CIF group, those in the GIF group had lower mean stool consistency scores at 2 weeks (GIF 4.69 (SD 1.44) v. CIF 5.46 (SD 0.96), $P<0.0001$ ) and 1 month (GIF 4.95 (SD 1.35) v. CIF 5.35 (SD 1.19), $P=0.01$ ). No differences were observed in the stool consistency scores at other assessment time points.

There were no differences in the mean length of each sleep episode or the total number of sleep episodes between the two formula-fed groups, with the exception that infants in the GIF group had a shorter mean length of each sleep episode in the evening (GIF 103 (sD 63) $v$. CIF 127 (sD 65) min, $P=0 \cdot 007$ ) and a longer mean length of each sleep episode at night (GIF 317 (SD 96) $v$. CIF 288 (SD 102) min, $P=0.03$ ) at the 2 -month assessment time point. The time taken to settle for sleep during the day, in the evening or at night also did not differ between the GIF and CIF groups. In comparison with the breast-fed infants, there were some differences in sleeping patterns between the formula-fed and breast-fed infants, but the differences were inconsistent (data not shown).

\section{Discussion}

The present study is the first to rigorously evaluate in healthy term infants the effect of feeding a goat infant formula up to 12 months of age on growth, nutritional status, tolerance to formula, and a wide range of health- and allergy-related outcomes in a well-conducted randomised controlled trial involving a control group fed a cow milk infant formula and a reference group of breast-fed infants. We could detect no difference in $z$-scores for infant weight, length, head circumference and weight for length up to 12 months between the two formula-fed groups. The same overall treatment effects

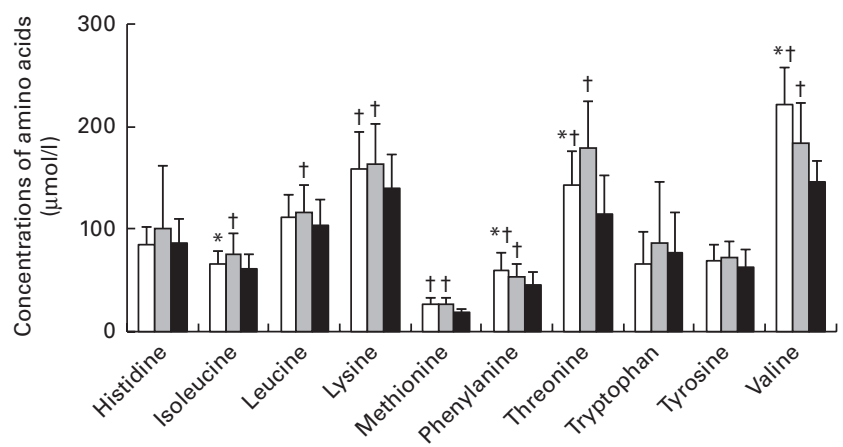

Fig. 3. Concentrations of essential and semi-essential amino acids in the plasma of infants after 4 months of being fed goat milk formula $\square$, cow milk formula $\square$ or breast milk $\square$. Values are means, with standard deviations represented by vertical bars. * Mean value was significantly different from that of the cow milk formula-fed group $(P<0.05)$. † Mean value was significantly different from that of the breast milk-fed group $(P<0.05)$. 
were observed in the intention-to-treat or per-protocol analysis that excluded data obtained from infants who consumed any non-study formula, liquids or solids for more than $12 \mathrm{~d}$ before 4 months of age. This suggests that it is unlikely that the consumption of non-study foods by some infants within the first 4 months had a significant impact on the outcomes of the study. We did detect some differences in weight and weight-for-length $z$-scores for both the formula-fed groups compared with the breast-fed group, consistent with the findings of other studies comparing the growth of formula and breast-fed infants ${ }^{(25-27)}$. Interestingly, while the differences in weight or weight-for-length $z$-scores persisted at 12 months between the breast-fed infants and cow milk formula-fed infants in the present study, consistent with the findings of other cow milk-based formula studies ${ }^{(25-27)}$, there was no differences between the goat milk formula-fed infants and breast-fed infants. The present study used the same formula with a lower protein content $(2 \mathrm{~g} / 418 \mathrm{~kJ}(100 \mathrm{kcal})$ and $2 \cdot 1 \mathrm{~g} / 418 \mathrm{~kJ}(100 \mathrm{kcal})$ for goat and cow milk formulas, respectively) through to 12 months rather than switching to a follow-on formula with a higher protein content from 6 months as has been done in the other formula studies ${ }^{(25-27)}$. This may partly explain the difference observed between the present study and the other formula studies mentioned above, as it has been shown that weight-for-length $z$-score at 24 months of infants fed a low-protein formula was not different from that of breast-fed infants, while infants fed a high-protein formula $(2 \cdot 9 \mathrm{~g} / 418 \mathrm{~kJ}(100 \mathrm{kcal}))$ had a higher $z$-score.

There were minor differences in the blood biomarkers between the formula-fed groups, which probably reflected differences in the composition of the two formulas. For instance, the cow infant formula contained added folate close to the recommended maximum, compared with the goat milk formula that had an amount in the mid-range of the recommendations ${ }^{(9,10)}$. Nevertheless, concentrations of blood biomarkers measured at 4 months were within the normal reference range for infants of this age ${ }^{(24)}$.

Whey proteins are often added to formulas to help improve the protein quality and availability of essential and semiessential amino acids ${ }^{(28,29)}$. Infant formulas made from goat milk without added whey proteins have been shown to have sufficient quantities of all the essential and semi-essential amino acids $^{(8)}$ and to have amino acid digestibility and absorption properties similar to a whey-based cow infant formula in an animal model ${ }^{(11)}$. The present study demonstrates some differences in plasma amino acid profile between the formula-fed groups as well as in comparison with the breast-fed infants, but there were large inter-individual variations. Although the differences were statistically significant, they are unlikely to be clinically important, as the mean plasma amino acid concentrations in infants in both the formula-fed groups are similar to those reported in other studies ${ }^{(30,31)}$.

The present study is the first to record a wide range of outcomes related to general health, gastrointestinal function and allergy when infants were exposed to a goat infant formula using a combination of objective clinical assessments and subjective parental reports. There were no differences in 
the objective assessments of allergy-related outcomes including dermatitis and medically diagnosed food allergy.

The only statistically significant finding between the formula-fed groups was a greater number of parental reports of blood-stained stools in infants fed the goat infant formula than in those fed the cow infant formula. We are unsure about the significance of this finding. First, the number of reports of blood-stained stools was low overall, and second, there was no indication of other gastrointestinal disorders, differences in stool characteristics, crying and sleeping patterns, general health or other allergy-related symptoms. Furthermore, none of the infants in the study had Fe-deficiency anaemia, which would indicate no significant blood loss over time. Finally, the outcomes related to allergy and gastrointestinal function were secondary outcomes, which the study did not have adequate power to rigorously assess, and thus they need to be interpreted with caution, as it is possible that this may be due to chance. A much larger, adequately powered randomised controlled trial with objective assessment of clinical outcomes and biomarkers of allergy is needed to rigorously evaluate the effects of goat milk infant formula on allergy and gastrointestinal function.

In conclusion, the growth and blood biomarkers of nutritional status of infants fed a whole-goat milk-based infant formula did not differ from those of infants fed a standard cow infant formula with added whey. The lack of a significant difference between the formula-fed groups for an extensive range of health-related outcomes and for the occurrence of serious adverse events supports the safety of using goat milk in infant formula.

\section{Acknowledgements}

The authors thank the families who participated in the study, the medical, nursing and research staff at each participating centre, the staff of the Child Nutrition Research Centre, and the staff of the Data Management and Analysis Centre, University of Adelaide and University of California, Davis, USA. M. M. and R. A. G. were supported by the National Health and Medical Research Council Senior Research Fellowship (ID: 565000 for M. M. and ID: 519324 for R. A. G.). Infrastructure support was provided by the Women's and Children's Health Research Institute, the University of Adelaide, the Women's and Children's Hospital Adelaide, the Flinders Medical Centre Adelaide and the Lyell McEwin Hospital Adelaide.

Dairy Goat Co-operative (N.Z.) Limited, New Zealand, provided the funding to conduct the study. The funder contributed to the design of the study, interpretation of the findings and preparation of the manuscript. Data collection, management and analysis were conducted independently of the funder.

The authors' contributions were as follows: M. M., S. J. Z., R. A. G., T. S., C. G. P. and D. J. L. designed the research; M. M., S. J. Z., R. A. G. and B. L. conducted the research; T. S., S. J. Z. and M. M. analysed the data and carried out the statistical analyses; S. J. Z. drafted the manuscript with contributions from all authors; M. M. and S. J. Z. had primary responsibility for the final content. All authors reviewed and approved the final manuscript.
M. M. serves on scientific advisory boards for Nestlé, Fonterra and Nutricia. R. A. G. serves on scientific advisory board for Fonterra. Associated honoraria for M. M. and R. A. G. are paid to their institutions to support conference travel and continuing education for postgraduate students and early career researchers. C. G. P. and D. J. L. work for the Dairy Goat Co-operative (N.Z.) Limited, which manufactured the goat milk formula used in the study. None of the other authors has any conflicts of interest to declare.

\section{References}

1. AAP (2012) Breastfeeding and the use of human milk. Pediatrics 129, e827-e841.

2. Raiha NC, Fazzolari-Nesci A, Cajozzo C, et al. (2002) Whey predominant, whey modified infant formula with protein/ energy ratio of $1.8 \mathrm{~g} / 100 \mathrm{kcal}$ : adequate and safe for term infants from birth to four months. I Pediatr Gastroenterol Nutr 35, 275-281.

3. Hernell O (2011) Human milk vs. cow's milk and the evolution of infant formulas. Nestle Nutr Workshop Ser Pediatr Program 67, 17-28.

4. Ziegler DS, Russell SJ, Rozenberg G, et al. (2005) Goats' milk quackery. J Paediatr Child Health 41, 569-571.

5. Basnet S, Schneider M, Gazit A, et al. (2010) Fresh goat's milk for infants: myths and realities - a review. Pediatrics 125 , e973-e977.

6. Taitz LS \& Armitage BL (1984) Goats' milk for infants and children. Br Med J (Clin Res Ed) 288, 428-429.

7. Baur LA \& Allen JR (2005) Goat milk for infants: yes or no? $J$ Paediatr Child Health 41, 543.

8. Rutherfurd S, Moughan P, Lowry D, et al. (2008) Amino acid composition determined using multiple hydrolysis times for three goat milk formulations. Int J Food Sci Nutr 59, 679-690.

9. Koletzko B, Baker S, Cleghorn G, et al. (2005) Global standard for the composition of infant formula: recommendations of an ESPGHAN coordinated international expert group. J Pediatr Gastroenterol Nutr 41, 584-599.

10. Codex Alimentarius Commission (2007) Standard for infant formula and formulas for special medical purposes intended for infants CODEX STAN 72-1981 (amended 2007). http:// www.codexalimentarius.org/input/download/standards/288/ CXS_072e.pdf (accessed March 2013).

11. Rutherfurd SM, Darragh AJ, Hendriks WH, et al. (2006) True ileal amino acid digestibility of goat and cow milk infant formulas. J Dairy Sci 89, 2408-2413.

12. Koletzko B, Ashwell M, Beck B, et al. (2002) Characterisation of infant food modifications in the European Union. Ann Nutr Metab 46, 231-242.

13. Silanikove N, Leitner G, Merin U, et al. (2010) Recent advances in exploiting goat's milk: quality, safety and production aspects. Small Rum Res 89, 110-124.

14. Razafindrakoto O, Ravelomanana N, Rasolofo A, et al. (1994) Goat's milk as a substitute for cow's milk in undernourished children: a randomised double-blind clinical trial. Pediatrics 94, 65-69.

15. Haenlein GFW (2004) Goat milk in human nutrition. Small Rum Res 51, 155-163.

16. Grant C, Rotherham B, Sharpe S, et al. (2005) Randomized, double-blind comparison of growth in infants receiving goat milk formula versus cow milk infant formula.J Paediatr Child Health 41, 564-568.

17. EFSA Panel on Dietetic Products Nutrition and Allergies (2004) Scientific opinion on the suitability of goat milk 
protein as a source of protein in infant formulae and in follow-on formulae. EFSA J 30, 1-15.

18. Koletzko B, von Kries R, Closa R, et al. (2009) Lower protein in infant formula is associated with lower weight up to age $2 \mathrm{y}$ : a randomised clinical trial. Am J Clin Nutr 89, 1836-1845.

19. Bang H, Ni L \& Davis CE (2004) Assessment of blinding in clinical trials. Control Clin Trials 25, 143-156.

20. European Task Force on Atopic Dermatitis (1993) Severity scoring of atopic dermatitis: the SCORAD index. Dermatology 186, 23-31.

21. Lewis SJ \& Heaton KW (1997) Stool form scale as a useful guide to intestinal transit time. Scand J Gastroenterol 32, 920-924.

22. Matthey S (2001) The sleep and settle questionnaire for parents of infants: psychometric properties. I Paediatr Child Health 37, 470-475.

23. World Health Organisation (2006) WHO child growth standards based on length/height, weight and age. Acta Paediatr Suppl 450, 76-85.

24. Himes R \& Shulman R (2008) Use of laboratory measurements in nutritional assessment. In Pediatric Nutrition in Practice, pp. 27-30 [B Koletzko, editor]. Basel: Karger.

25. Kramer MS, Guo T, Platt RW, et al. (2004) Feeding effects on growth during infancy. J Pediatr 145, 600-605.

26. Dewey KG, Heinig MJ, Nommsen LA, et al. (1992) Growth of breast-fed and formula-fed infants from 0 to 18 months - the DARLING Study. Pediatrics 89, 1035-1041.
27. Agostoni C, Grandi F, Gianni ML, et al. (1999) Growth patterns of breast fed and formula fed infants in the first 12 months of life: an Italian study. Arch Dis Child 81, 395-399.

28. Janas LM, Picciano MF \& Hatch TF (1987) Indices of protein metabolism in term infants fed either human milk or formulas with reduced protein concentration and various whey casein ratios. J Pediatr 110, 838-848.

29. Janas LM, Picciano MF \& Hatch TF (1985) Indices of protein metabolism in term infants fed human milk, whey predominant formula or cow's milk formula. Pediatrics $\mathbf{7 5}$ $775-784$

30. Lonnerdal B \& Hernell O (1998) Effects of feeding ultrahigh-temperature (UHP)-treated infant formula with different protein concentrations or powdered formula, as compared with breast-feeding, on plasma amino acids, hematology, and trace element status. Am J Clin Nutr 68, $350-356$

31. Sandstrom O, Lonnerdal B, Graverholt G, et al. (2008) Effects of alpha-lactalbumin-enriched formula containing different concentrations of glycomacropeptide on infant nutrition. Am J Clin Nutr 87, 921-928.

32. Wijesinha-Bettoni R \& Burlingame B (2013) Milk and dairy product composition. In Milk and Dairy Products in Human Nutrition, Chapter 3, pp. 41-90 [E Muehlhoff, A Bennett and D McMahon, editors]. Rome, Italy: FAO. 